\title{
Apports en acides gras polyinsaturés, notamment en DHA, dans la population française adulte : données issues de l'étude SU.VI.MAX et comparaisons avec d'autres études'
}

\author{
Pierre ASTORG \\ Unité Nutrition et régulation lipidique \\ des fonctions cérébrales (Nu.Ré.Li.Ce), \\ INRA, Centre de Recherche de Jouy, \\ Domaine de Vilvert, bât. 230, \\ 78352 Jouy-en-Josas cedex \\ <pierre.astorg@jouy.inra.fr>
}

\begin{abstract}
The intake of individual $n-6$ and $n-3$ polyunsaturated fatty acids (PUFA) have been estimated in 4,884 adult subjects of both sexes, volunteers from the French SUVIMAX intervention trial. On an energy basis, both men and women consumed $4,2 \%$ energy as linoleic acid, $0,38 \%$ as $\alpha$-linolenic acid, $0,08 \%$ as arachidonic acid, $0,06 \%$ as eicosapentaenoic acid (EPA), 0,03\% as docosapentaenoic acid and $0,11 \%$ as docosahexaenoic acid (DHA), with a mean linoleic/ $\alpha$-linolenic acid ratio of 11,3 . The intake of $\alpha$-linolenic acid was well below the current recommendations for almost all subjects, as a consequence of the low consumption of $\alpha$-linolenic acid-rich oils and fats. The mean intake levels of long-chain n-3 PUFA, especially of DHA, were higher than the recommended levels, but showed great interindividual variations, due to very large differences in the consumption of fish, especially of fatty fish.
\end{abstract}

Key words: dietary intake, linoleic acid, arachidonic acid, $\alpha$-linolenic acid, long-chain n-3 PUFA, EPA, DHA
Les acides gras essentiels sont des nutriments de première importance pour le développement et la santé de l'homme, et les recherches des 15 dernières années ont demontré le rôle d'un apport adéquat en acides gras polyinsaturés (AGPI) en $\mathrm{n}$ - 3 dans la prévention de nombreuses maladies, en particulier des maladies cardiovasculaires [1]. L'acide linoléique (18:2 n-6) et l'acide $\alpha$-linolénique (18:3n-3), précurseurs d'origine végétale des AGPI n-6 et $n-3$, respectivement, sont en compétition pour les mêmes enzymes qui les convertissent en AGPI à 20 et 22 atomes de carbone : en conséquence, leurs niveaux d'apport optimaux sont mutuellement dépendants. Dans les pays occidentaux, l'utilisation croissante au cours des 50 dernières années d'huiles végétales riches en acide linoléique et pauvres en acide $\alpha$-linolénique dans l'alimentation animale et humaine a abouti à l'augmentation du rapport n-6/n-3 (ou linoléique/a-linolénique) dans le régime alimentaire des populations, au-dessus des valeurs considérées comme optimales [2-4]. L'évaluation des apports en acides gras essentiels d'une population implique l'estimation des apports tant des précurseurs (acides linoléique et $\alpha$-linolénique) que des AGPI à longue chaîne des séries $n-6$ et $n-3$. En effet, la conversion des acides linoléique et $\alpha$-linolénique en AGPI à longue chaîne n'a lieu chez l'homme qu'avec un rendement faible [5], l'ingestion même de faibles quantités

\footnotetext{
${ }^{1}$ Une grande partie des données citées dans cet article a été publiée dans Lipids 2004 ; 39 : 527-535, sous le titre: "Dietary intakes and food sources of n-6 and n-3 PUFA in French adult men and women ».
}

d'AGPI à longue chaîne peut contribuer significativemment au statut en AGPI [4]. Au cours des dernières années, des apports recommandés ont été publiés dans plusieurs pays, dont la France, non seulement pour les acides linoléique et $\alpha$-linolénique, mais aussi pour les AGPI à longue chaîne, notamment pour l'acide docosahexaenoïque (DHA) $[6,7]$. Cependant, on manque encore de données sur les niveaux d'apports réels des différents AGPI n-6 et n-3 dans les populations. En particulier, les travaux ayant mesuré les apports en AGPI à longue chaîne sont peu nombreux, car ils dépendent de l'existence et de la qualité des tables des teneurs des aliments en AGPI individuels. La première étude concernant la population française a estimé les apports en acides linoléique and $\alpha$-linolénique [8]. Des travaux récents ont estimé la consommation d'AGPI à longue chaîne dans des pays d'Europe et d'Amérique du Nord, ainsi qu'en Australie et au Japon [9-18]. Nous présentons ici des données issues de l'étude SU.VI.MAX, une étude d'intervention prospective sur un échantillon d'adultes français des deux sexes [19], et aussi de la cohorte E3N-EPIC [20] et de l'étude INCA1 [21].

\section{Matériel and méthodes}

\section{Échantillon de population}

L'étude SU.VI.MAX est un essai d'intervention primaire randomisé dont le but principal était de tester les effets d'une supplémentation quotidienne par un mélange de minéraux et de vitamines sur l'incidence de maladies cardiovasculaires et de cancers dans une population d'adultes des deux sexes en France [19]. Un autre objectif de l'étude était d'évaluer la consommation alimentaire de la population française adulte. L'échantillon de population consistait en 12741 sujets (5 028 hommes et 7713 femmes) de toutes les régions de France, recrutés en 1994-1995 en vue d'un suivi de 8 ans. Les hommes étaient âgés de 45 à 63 ans et les femmes de 35 à 63 ans à l'inclusion. D'autres détails sur le plan de l'étude, le recrutement, et les caractéristiques des sujets au départ ont été publiés précédemment [19].

\section{Questionnaire alimentaire}

À l'inclusion et tous les deux mois jusqu'à la fin de l'étude, les sujets devaient remplir un questionnaire portant sur un enregistrement alimentaire de 24 heures, c'est-à-dire six questionnaires par an. Un grand choix d'aliments et de boissons (environ 900 items) était offert pour chacun des trois repas et pour 4 autres occasions de prise d'aliments ou de boissons. Les sujets devaient indiquer la taille des portions consommées, en s'aidant d'un manuel de photographies remis au début de l'étude. Le questionnaire comportait aussi des questions sur les méthodes de cuisson, et sur les corps gras utilisés pour la cuisson et l'assaisonnement. Pour cette étude, nous avons sélectionné les participants ayant rempli au moins 10 questionnaires dans une période de 2,5 ans entre l'inclusion et 1998. On a montré précédemment que 10 enregistrements de 24 heures suffisent pour estimer la consommation individuelle des principales classes d'acides gras (saturés, mono-insaturés, et AGPI) avec une bonne précision. 


\section{Table de composition des aliments}

Une table de composition adaptée à l'analyse des données alimentaires collectées dans I'étude SU.VI.MAX a été développée [22]. Les teneurs des aliments en énergie, en lipides totaux, en acides linoléique, $\alpha$-linolénique, arachidonique, et en AGPI n-3 à longue chaîne: eicosapentaénoïque (EPA), docosapentaénoïque (22:5n-3) et docosahexaénoïque (DHA), ont été déterminées sur la base de tables existantes: la table de composition des aliments française $[23,24]$; la table américaine US Department of Agriculture National Nutrient Database [25]; et la table britannique McCance \& Widdowson's Food Composition Table [26], sur des données fournies par I'Iterg, et sur la base de publications originales. En particulier, beaucoup de données sur les teneurs en AGPI des viandes, volailles, œufs, poissons et fruits de mer sont tirés de publications originales, les tables existantes étant souvent incomplètes.

\section{Résultats}

4884 sujets (2099 hommes et 2785 femmes), représentant $38,3 \%$ de l'ensemble de la cohorte $(41,7 \%$ des hommes et $36,1 \%$ des femmes) ont fourni des données alimentaires complètes provenant d'au moins 10 enregistrements de 24 heures. Les quantités ingérées de lipides totaux et des différents AGPI n-6 et n-3 figurent dans le tableau 1. Si on exprime les ingérés en \% de l'énergie, il apparaît que les femmes ont consommé sensiblement plus de lipides que les hommes $(38,1$ vs. $36,3 \%$ de l'énergie), mais il n'y a pas de différence entre les sexes en ce qui concerne la consommation d'AGPI. Les apports en acides linoléique et $\alpha$-linolénique repésentaient $4,2 \%$ et $0,38 \%$ de l'énergie, avec un rapport linoléique/ $\alpha$ linolénique élévé : 11,3 en moyenne, au-dessus de 7,6 pour $95 \%$ de la population. La moitié de l'échantillon (56,8\% des hommes et $45,6 \%$ des femmes) avait à la fois un apport en acide $\alpha$-linolénique inférieur à $0,4 \%$ de l'énergie et un rapport linoléique/ $\alpha$-linolénique supérieur à 10 . Alors que l'apport moyen en acide arachidonique était de 0,08 \% de l'énergie dans les deux sexes, celui des AGPI n-3 à longue chaîne représentait $0,21 \%$ de l'énergie, à raison de $55 \%$ de DHA, $30 \%$ d'EPA et $15 \%$ de $22: 5$ n-3. À la différence des autres AGPI, les apports en AGPI n-3 à longue chaîne, en particulier ceux d'EPA et de DHA, montrent une distribution asymétrique très étirée. Le rapport entre le $95^{\mathrm{e}}$ et le $5^{\mathrm{e}}$ percentile est d'environ 15 pour l'EPA et 10 pour le DHA, au lieu de 2 à 3 pour les acides linoléique, $\alpha$-linolénique and arachidonique : les variations individuelles des apports en EPA et en DHA sont nettement plus grandes que pour les autres AGPI. Pour $50 \%$ de l'échantillon, l'apport en acide linoléique est supérieur à l'ANC (4\% de l'apport énergétique total ou $\mathrm{AET}$ ), et $95 \%$ ont un apport supérieur aux deux tiers de I'ANC (besoin nutritionnel moyen, BNM). Pour I'acide $\alpha$-linolénique, ces proportions tombent à $0,1 \%$ et $6 \%$, respectivement (ANC : $0,8 \%$ de l'AET), alors qu'elles atteignent $78 \%$ et
$90 \%$ pour le DHA (ANC : 0,05\% de l'AET), $95 \%$ de la population ayant un apport en DHA égal ou supérieur à la moitié de l'ANC.

Les apports (en g/jour) en acides linoléique, en acide $\alpha$-linolénique, en acide arachidonique et en 22:5 n-3 sont liés aux apports en énergie et en lipides totaux (tableau 2), la corrélation étant la plus forte avec l'acide $\alpha$-linolénique. De plus, à apport énergétique constant, des apports croissant en acide $\alpha$-linolénique sont corrélés à des apports croissants de lipides. Les apport en acides linoléique et $\alpha$-linolénique sont fortement corrélés avec la consommation de viande, abats, volaille et œufs, alors que seul l'apport en acide linoléique est corrélé à la consommation d'huiles végétales. L'apport en acide arachidonique est très corrélé avec la consommation de viande, abats, volailles et oeufs, alors que les apports en EPA et en DHA sont fortement corrélés avec la consommation de poisson et de fruits de mer. Enfin, l'apport en 22:5 n-3 est corrélé à la fois avec la consommation de poisson et de fruits de mer et avec la consommation de viande, abats, volailles et œufs.

Les contributions en pourcentage des différents aliments aux apports en chacun des AGPI sont pratiquement les mêmes dans les deux sexes, et les résultats sont donnés pour l'ensemble de l'échantillon (figure 1). Les corps gras sont la principale source d'acide linoléique, et contribuent à un tiers de l'apport. Parmi les corps gras, les huiles végétales sont de loin la principale source, les margarines ne contribuant que pour une faible part à l'apport en

Tableau 1. Apports en lipides totaux et en AGPI n-6 et n-3 ( $g$ or mg/jour).

\begin{tabular}{|c|c|c|c|c|c|c|c|c|c|}
\hline & $\begin{array}{l}\text { Lipides totaux } \\
(\mathrm{g} / \mathrm{j})\end{array}$ & $\begin{array}{c}18 \text { :2 n-6 } \\
(\mathrm{g} / \mathrm{j})\end{array}$ & $\begin{array}{c}18 \text { :3 n-3 } \\
(\mathrm{g} / \mathrm{j})\end{array}$ & $\begin{array}{c}18: 2 n-6 / \\
18: 3 n-3\end{array}$ & $\begin{array}{c}20: 4 \text { n-6 } \\
(\mathrm{mg} / \mathrm{j})\end{array}$ & $\begin{array}{c}20: 5 \text { n-3 } \\
(\mathrm{mg} / \mathrm{j})\end{array}$ & $\begin{array}{c}22: 5 \text { n-3 } \\
(\mathrm{mg} / \mathrm{j})\end{array}$ & $\begin{array}{c}22 \text { :6 n-3 } \\
(\mathrm{mg} / \mathrm{j})\end{array}$ & $\begin{array}{c}\mathbf{L C} \mathbf{n}-\mathbf{3}^{\mathrm{a}} \\
(\mathrm{mg} / \mathrm{j})\end{array}$ \\
\hline \multicolumn{10}{|l|}{$\begin{array}{l}\text { Hommes } \\
(n=2099):\end{array}$} \\
\hline moyenne & 94,06 & 10,64 & 0,94 & 11,5 & 203,9 & 149,9 & 74,8 & 272,6 & 497,3 \\
\hline écart-type & 22,42 & 3,28 & 0,25 & 3,0 & 66,0 & 112,1 & 33,0 & 191,3 & 325,1 \\
\hline minimum & 32,11 & 2,74 & 0,37 & 4,8 & 55,1 & 4,5 & 12,9 & 10,0 & 39,9 \\
\hline $5^{\text {th }}$ percentile & 59,97 & 6,13 & 0,59 & 7,7 & 111,1 & 27,5 & 33,5 & 66,0 & 139,3 \\
\hline médiane & 93,32 & 10,12 & 0,92 & 11,0 & 197,0 & 119,6 & 68,2 & 221,3 & 408,3 \\
\hline $95^{\text {th }}$ percentile & 134,42 & 16,74 & 1,37 & 16,9 & 323,2 & 375,1 & 138,4 & 668,4 & 1159,3 \\
\hline maximum & 187,53 & 27,85 & 2,62 & 39,3 & 558,2 & 768,5 & 275,7 & 1472,8 & 2617,0 \\
\hline \multicolumn{10}{|l|}{$\begin{array}{l}\text { Femmes } \\
(n=2785):\end{array}$} \\
\hline moyenne & 73,55 & 8,10 & 0,74 & 11,1 & 151,9 & 117,8 & 55,9 & 225,9 & 399,6 \\
\hline écart-type & 19,17 & 2,60 & 0,20 & 2,7 & 49,2 & 94,2 & 27,8 & 170,9 & 286,0 \\
\hline minimum & 19,46 & 2,10 & 0,21 & 4,8 & 14,5 & 2,0 & 2,3 & 6,5 & 10,8 \\
\hline $5^{\text {th }}$ percentile & 44,29 & 4,29 & 0,45 & 7,6 & 79,2 & 19,2 & 22,9 & 50,0 & 101,3 \\
\hline médiane & 72,00 & 7,80 & 0,71 & 10,7 & 147,8 & 91,4 & 50,2 & 177,0 & 320,6 \\
\hline $95^{\text {th }}$ percentile & 106,88 & 12,75 & 1,10 & 16,3 & 238,6 & 308,5 & 109,1 & 574,2 & 980,2 \\
\hline maximum & 172,15 & 21,11 & 1,98 & 32,1 & 34,4 & 853,3 & 269,5 & 1770,2 & 2893,0 \\
\hline
\end{tabular}

${ }^{a}$ AGPI n-3 à longue chaîne : somme de $20: 5$ n-3, $22: 5$ n-3 et $22: 6$ n-3. 
Table 2. Apports en AGPI n-6 et n-3: corrélations avec les apports en énergie, lipides totaux et quelques groupes d'aliments (coefficients de corrélation de Spearman).

\begin{tabular}{|c|c|c|c|c|c|c|c|}
\hline & $18: 2 n-6$ & $18: 3 n-3$ & $20: 4 n-6$ & $20: 5 n-3$ & $22: 5 n-3$ & $22: 6 n-3$ & LC $n-3^{2}$ \\
\hline $18: 3 n-3$ & $0,73^{b}$ & & & & & & \\
\hline $20: 4$ n-6 & 0,54 & 0,53 & & & & & \\
\hline $20: 5 n-3$ & 0,18 & 0,20 & 0,36 & & & & \\
\hline $22: 5 n-3$ & 0,39 & 0,39 & 0,70 & 0,80 & & & \\
\hline $22: 6 n-3$ & 0,20 & 0,21 & 0,40 & 0,93 & 0,81 & & \\
\hline LC n-3 & 0,22 & 0,23 & 0,44 & 0,97 & 0,86 & 0,99 & \\
\hline Energie & 0,69 & 0,83 & 0,57 & 0,20 & 0,42 & 0,21 & 0,24 \\
\hline Lipides totaux & 0,74 & 0,85 & 0,63 & 0,19 & 0,44 & 0,21 & 0,24 \\
\hline Poissons gras \& demi-gras ${ }^{c}$ & 0,11 & 0,12 & 0,24 & 0,83 & 0,67 & 0,85 & 0,85 \\
\hline Poissons \& fruits de mer ${ }^{d}$ & 0,17 & 0,18 & 0,27 & 0,75 & 0,54 & 0,73 & 0,74 \\
\hline Viandes, volailles \& œuff $\mathrm{e}^{\mathrm{e}}$ & 0,48 & 0,45 & 0,75 & 0,11 & 0,50 & 0,10 & 0,16 \\
\hline Produits laitiers & $\begin{array}{c}0,04 \\
p=0,006\end{array}$ & 0,16 & $\begin{array}{l}-0,01 \\
p>0,1\end{array}$ & $\begin{array}{c}0,01 \\
p>0,1\end{array}$ & $\begin{array}{l}-0,02 \\
p>0,1\end{array}$ & $\begin{array}{c}0,02 \\
p>0,1\end{array}$ & $\begin{array}{c}0,01 \\
p>0,1\end{array}$ \\
\hline Huiles végétales & 0,53 & 0,29 & 0,26 & 0,11 & 0,22 & 0,10 & 0,12 \\
\hline Huiles de colza, de soja \& de noix & 0,05 & 0,11 & $\begin{array}{c}0,00 \\
p>0,1\end{array}$ & 0,05 & $\begin{array}{c}0,03 \\
p=0,04\end{array}$ & 0,05 & 0,05 \\
\hline
\end{tabular}

${ }^{a}$ AGPI n-3 à longue chaîne : somme de $20: 5$ n-3, $22: 5$ n-3 et $22: 6$ n-3.

${ }^{b}$ Tous les coefficients de corrélation sont significatifs pour $p<0,001$, sauf ceux où une valeur de $p$ est indiquée.

' Poissons gras (plus de 1,5 g d'AGPI n-3 à longue chaîne/100 g) : œufs de poisson, hareng, maquereau, saumon, sardines fraîches, thon frais ; poissons demi-gras (entre $0,5 \mathrm{~g}$ et 1,5 g d'AGPI n-3 à longue chaîne/100 g) : anchois, anguille, bar, carpe, flétan, mulet, saumonette, sardines en boîte, truite ; poissons maigres : les autres espèces de poissons mentionnées (moins de 0,5 g d'AGPI n-3 à longue chaîne/100 g).

${ }^{\mathrm{d}}$ Comprend les poissons gras, les poissons demi-gras, les poissons maigres et les fruits de mer.

e Comprend la viande, la charcuterie, les abats, la volaille, le gibier et les œufs.

acide linoléique. Les produits d'animaux terrestres autres que les produits laitiers sont aussi une source importante d'acide linoléique. Presque tous les types d'aliments, sauf les poissons et les fruits de mer, contribuent de façon significative à l'apport en acide $\alpha$-linolénique. Les produits laitiers et les viandes, volailles, abats et œufs sont des sources majeures, fournissant ensemble plus de $41 \%$ de l'apport. Les corps gras fournissent seulement $10 \%$ de l'apport en acide $\alpha$-linolénique, car les huiles riches en cet acide gras (colza, soja, noix) ne sont que faiblement consommées en France : les huiles végétales consommées dans l'échantillon de population sont l'huile de tournesol (38,5\% des quantités d'huiles végétales consommées), I'huile d'olive (35,2\%), l'huile d'arachide $(13,3 \%)$, les huiles de mélange $(8,0 \%)$, I'huile de pépin de raisin $(1,6 \%)$, l'huile de mais $(1,6 \%)$, I'huile de colza $(0,9 \%)$, I'huile de noix

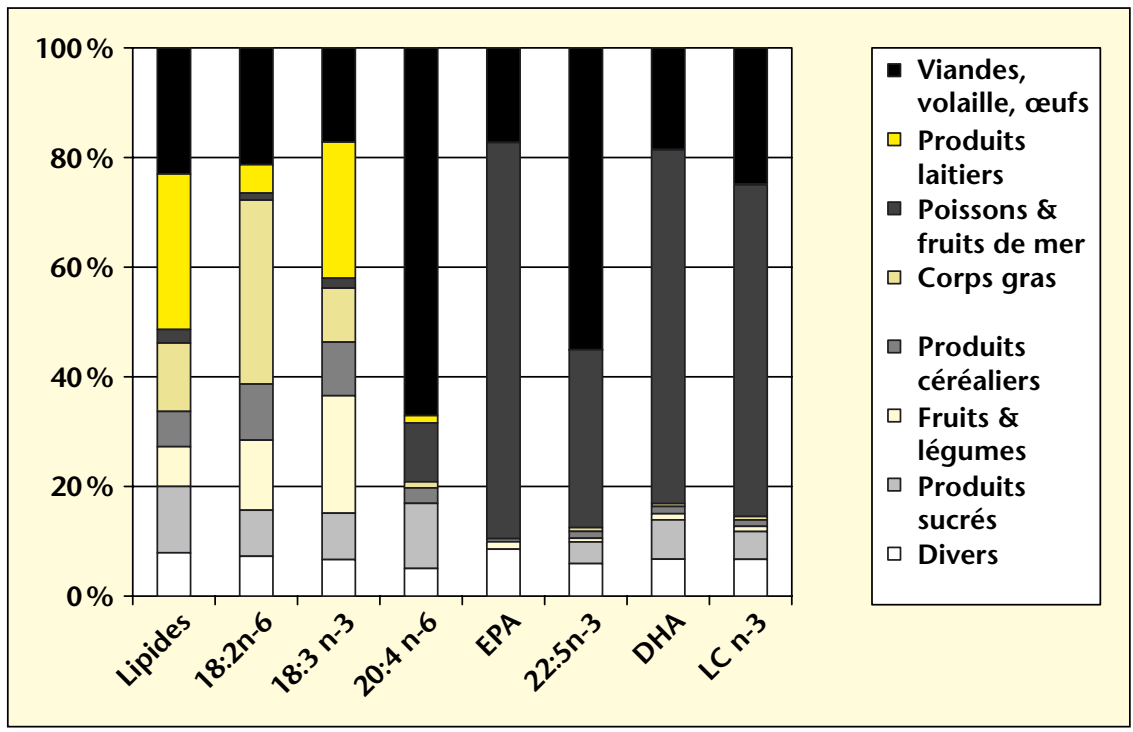

Figure 1. Contributions des familles d'aliments aux apports en lipides totaux et en acides gras polyinsaturés (linoléique, $\alpha$-linolénique, arachidonique, EPA, $22: 5 n$-3, DHA). (\% de l'apport total).
$(0,6 \%)$ et l'huile de soja $(0,3 \%)$, soit moins de $2 \%$ du total pour les huiles riches en acide $\alpha$-linolénique. Les viandes, volailles, abats et œufs sont de loin la principale source d'acide arachidonique, contribuant à plus des deux tiers de l'apport. Les principales sources d'AGPI n-3 à longue chaîne sont les poissons et fruits de mer, et les produits animaux autres que les produits laitiers (figure 1). Les poissons et les fruits de mer contribuent à $72 \%$ et $65 \%$, respectivement, de l'apport en EPA et en DHA, les poissons gras (harengs, sardines, maquereaux, saumon, thon frais) fournissant à eux seuls $33 \%$ de I'EPA et du DHA, et $31 \%$ du total des AGPI n-3 à longue chaîne. Le 22:5 n-3 est principalement apporté par les viandes, les volailles et les œufs, qui contribuent à $55 \%$ de l'apport, les poissons et fruits de mer étant la seconde source (32\%). Bien que minoritaire, la contribution des viandes, volailles, abats et œufs à I'apport en AGPI n-3 à longue chaîne est significative : en particulier, on peut estimer que, en moyenne, 20 à $25 \%$ du DHA ingéré proviennent de ces aliments : $54-68 \mathrm{mg} / \mathrm{jour}$ chez les hommes et $45-66 \mathrm{mg} / \mathrm{jour}$ chez les femmes, soit environ la moitié de l'ANC de DHA (120 mg/jour et $100 \mathrm{mg} /$ jour, respectivement). De grandes différences individuelles dans la consommation de poisson et de fruit de mer déterminent des écarts important dans les apports en AGPI n-3, notamment en DHA : les sujets situés dans le $5^{\mathrm{e}}$ quintile d'apport en AGPI n-3 à longue chaîne consomment 24 fois plus de poissons gras, 7 fois plus de poisson 


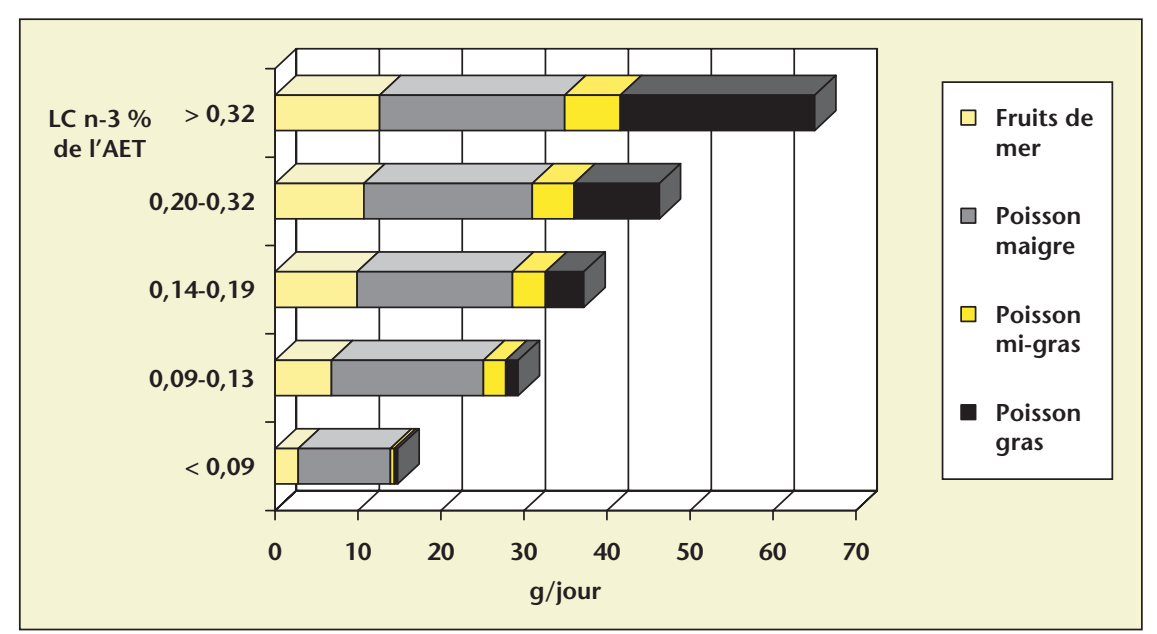

Figure 2. Consommation de poisson et de fruit de mer ( $g /$ jour) chez les femmes de la cohorte SU.VI.MAX en fonction de l'apport en AGPI n-3 à longue chaîne ( $L C n-3$ : somme EPA + $22: 5 n-3+D H A ; \%$ de l'apport énergétique total, quintiles).

demi-gras, deux fois plus de poisson maigre et 4 fois plus de fruits de mer que les sujets situés dans le premier quintile (figure 2). La consommation de poisson et fruits de mer varie de $15-20 \mathrm{~g} /$ jour, soit une portion par semaine, dans le premier quintile, à 65-80 g/jour, soit 4 portions par semaine, dans le cinquième ; il y a très peu de non-consommateurs de poisson.

\section{Discussion}

Les points forts de cette étude sont le recrutement national et la taille de l'échantillon, ainsi que la méthode utilisée pour mesurer les consommations: les enregistrements de 24 heures répétés. Sa principale limite réside dans le recrutement de volontaires d'une étude $d$ 'intervention, très vraisemblablement plus soucieux de leur santé et de leur alimentation que la moyenne des Français de la même tranche d'âge.

Les apports en AGPI en France ont été mesurés dans trois autres études: les femmes d'Aquitaine [8], la cohorte E3N, composée de femmes adhérentes à la MGEN [20], et l'étude INCA1, échantillon représentatif de la population française adulte des deux sexes [21]. Malgré des échantillons et des méthodes différentes, ces études donnent des résultats proches des nôtres pour l'estimation des apports en acides linoléique et $\alpha$-linolénique et de leur rapport, les valeurs plus élevées trouvées dans l'étude E3N étant en partie dues à un apport énergétique plus élevé (tableau 3). En ce qui concerne les apports en AGPI à longue chaîne, nos résultats et ceux de la cohorte $\mathrm{E} 3 \mathrm{~N}$ sont très proches, quasiment identiques si on les exprime en pourcentage de l'apport énergétique (AET) : 0,08-0,09\% pour l'acide arachidonique, 0,06$0,07 \%$ pour l'EPA, $0,03 \%$ pour le DPA, et
0,12-0,13\% pour le DHA. En revanche, des valeurs moyennes sensiblement plus basses pour I'EPA et le DHA sont trouvées dans l'étude INCA1 : $0,04 \%$ et $0,08 \%$ de l'AET, respectivement, soit 30 à $40 \%$ de moins, alors que les apports en acide arachidonique et en $22: 5 n-3$ sont sensiblement les mêmes que dans SU.VI. $M A X$ et $E 3 N$. Il est à noter que les études $E 3 N$ et INCA1 ont utilisé la table SU.VI.MAX, et que les valeurs plus basses trouvées dans INCA1 pour I'EPA et le DHA sont uniquement dues à de moindres apports, dus eux-mêmes à une proportion notable de non-consommateurs de poisson (20\%). Dans INCA1, 50 \% de la population atteint I'ANC du DHA (au lieu de $80 \%$ dans SU.VI.MAX). Des trois études citées, a apport énergétique total.
INCA1 est sans doute la plus représentative de la population française adulte.

Malgré de grandes différences dans les habitudes alimentaires, les apports en acide linoléique ne montrent pas de grandes variations entre des échantillons de population de divers pays (Europe, Amérique du Nord, Australie et Japon) : la plupart des estimations de l'apport en acide linoléique sont comprises entre 4 et $6 \%$ de l'apport énergétique [8-17]. En revanche, l'apport moyen en acide $\alpha$-linolénique varie très significativement entre populations : de 0,3 à $1 \%$ de l'apport énergétique. On trouve les apports moyens les plus élevés au Japon : 1,7-2,2 g/jour ou 0,7-1 \% de l'énergie, avec un rapport linoléique/ $\alpha$-linolénique compris entre 4 et $8[14,17,27-30]$, en conséquence de l'usage répandu des huiles de colza et de soja dans ce pays [31, 32]. Dans les pays occidentaux (États-Unis, Canada, Europe du Nord, Australie), l'apports moyen en $\alpha$-linolénique est de 0,5-0,6\% de l'apport énergétique, avec un rapport linoléique $/ \alpha$ linolénique compris entre 6 et $10[9-11,13,15$, $16,33,34]$. L'étude TRANSFAIR a comparé les apports en acides gras d'échantillons de populations de 14 pays d'Europe [35]. Dans cette étude, l'apport en $\alpha$-linolénique a été estimé à 0,5-0,8 g/jour chez les hommes dans les pays méditerranéens, dont la France, alors qu'il est beaucoup plus élevé dans les pays d'Europe du nord-ouest (1,4-2,5 g/jour) (figure 3), vraisemblablement en raison de la consommation beaucoup plus faible de margarines en France et en Europe du Sud [36].

Les estimations des apports en AGPI n-3 à longue chaîne montrent de grandes variations selon les études et les pays. Les apports les plus

Tableau 3. Apports en énergie (MJ/jour), en lipides totaux et en AGPI n-6 et n-3 ( $\mathrm{g} / \mathrm{jour}$ ) chez des femmes françaises adultes : comparaisons des études Femmes d'Aquitaine, SU.VI.MAX, E3N et INCA1 (moyennes \pm écarts-types).

\begin{tabular}{|c|c|c|c|c|}
\hline Etude & $\begin{array}{c}\text { Femmes } \\
\text { d'Aquitaine }\end{array}$ & SU.VI.MAX & E3N & INCA1 \\
\hline Effectif & $\mathbf{N}=79$ & $N=2785$ & $N=66823$ & $N=736$ \\
\hline $\begin{array}{l}\text { Méthode } \\
\text { de recueil } \\
\text { des données } \\
\text { alimentaires }\end{array}$ & Semainier & $\begin{array}{c}\text { Dix } \\
\text { enregistrements } \\
\text { de } 24 \text { h }\end{array}$ & $\begin{array}{l}\text { Questionnaire } \\
\text { de fréquence }\end{array}$ & Semainier \\
\hline $\mathrm{AET}^{\mathrm{a}} \mathrm{MI} / \mathrm{j}$ & $7,47 \pm 2,9$ & $7,19 \pm 1,80$ & $8,82 \pm 2,32$ & $7,80 \pm 1,53$ \\
\hline Lipides totaux & $83,7 \pm 23,7$ & $73,6 \pm 18,2$ & $88,8 \pm 27,6$ & $81,1 \pm 19,2$ \\
\hline $18: 2 n-6$ & $8,7 \pm 4,1$ & $8,1 \pm 2,6$ & $13,9 \pm 6,0$ & $9,8 \pm 3,6$ \\
\hline $18: 3 n-3$ & $0,6 \pm 0,2$ & $0,74 \pm 0,20$ & $1,02 \pm 0,37$ & $0,79 \pm 0,32$ \\
\hline $18: 2 n-6 / 18: 3 n-3$ & $15 \pm 8$ & $11,1 \pm 2,7$ & $14,2 \pm 5,8$ & $12,9 \pm 4,4$ \\
\hline $20: 4 n-6$ & - & $0,15 \pm 0,05$ & $0,21 \pm 0,09$ & $0,17 \pm 0.07$ \\
\hline $20: 5 n-3$ ou EPA & - & $0,12 \pm 0,09$ & $0,15 \pm 0,11$ & $0,08 \pm 0,10$ \\
\hline $22: 5 n-3$ & - & $0,06 \pm 0,03$ & $0,06 \pm 0,03$ & $0,06 \pm 0,03$ \\
\hline $22: 6 n-3$ ou DHA & - & $0,23 \pm 0,17$ & $0,29 \pm 0,19$ & $0,17 \pm 0,19$ \\
\hline $\mathrm{LC} n-3^{\mathrm{b}}$ & - & $0,40 \pm 0,29$ & $0,50 \pm 0,33$ & $0,31 \pm 0,33$ \\
\hline
\end{tabular}

${ }^{b}$ AGPI n-3 à longue chaîne : somme de $20: 5$ n-3 , 22 :5 n-3 et 22 :6 n-3. 


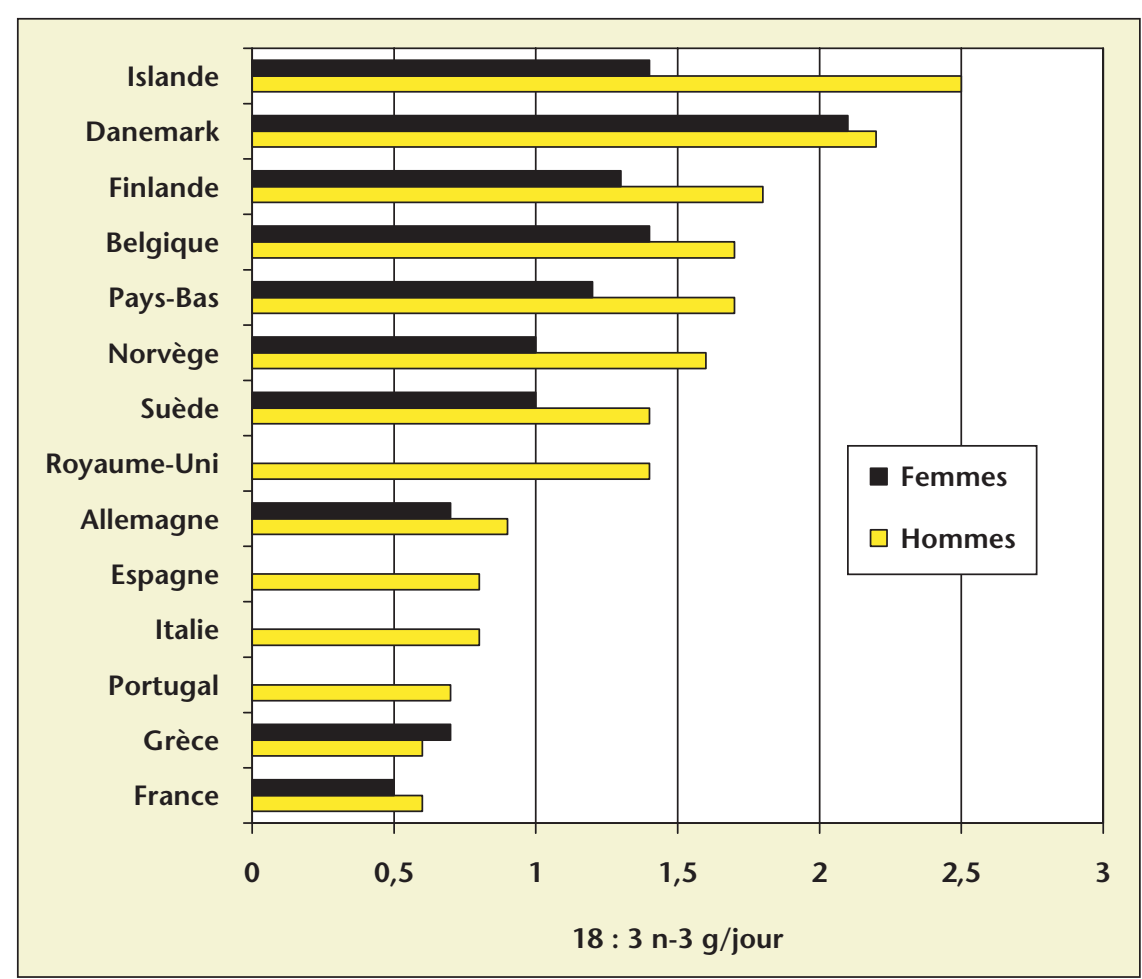

Figure 3. Apports moyens en acide $\alpha$-linolénique (g/jour) dans 14 pays d'Europe : étude TRANSFAIR (d'après Hulshof et al., réf. 34).

élevés sont observés au Japon: 1-1,5 g d'EPA+DHA /jour chez les hommes et 0,7-1,1 $\mathrm{g} /$ jour chez les femmes $[14,17,27,29,30]$, mais des valeurs élevées ont été trouvées en Norvège [12] et en Espagne [37]. Dans ces pays, l'apport en moyen DHA est de l'ordre de 0,7-1 g/jour (au Japon) ou 0,5-0,7 g/jour (en Norvège et en Espagne), ce qui s'explique par la plus grande consommation de poisson dans les pays scandinaves et en Espagne que dans le reste de l'Europe [38]. Les valeurs les plus basses d'apport en AGPI n-3 à longue chaîne ont été trouvées aux États-Unis [39], au Canada [11], en Belgique [18], en Allemagne [15] et en Australie $[10,16]$, I'apport en DHA étant alors de 0,1 à $0,2 \mathrm{~g} /$ jour (figure 4 ). La France se situe dans la zone intermédiaire $(0,4-0,5 \mathrm{~g} / \mathrm{jour}$ d'AGPI n-3 à longue chaîne en moyenne, dont 0,2-0,3 g de DHA), en raison d'une consommation moyenne de poisson et de fruits de mer parmi les plus élevées en Europe, après l'Espagne et les pays scandinaves [38] (figure 5). Chez les consommateurs réguliers de poisson des villes côtières de France, l'apport en DHA atteint 0,8 g/jour, comme au Japon [40] (figure 4).

\section{Conclusion}

En résumé, les études présentées montrent que, alors que l'apport en acide linoléique est population adulte. comparable à celui trouvé dans beaucoun a-linolénique dans la population française adulte est plus bas que ceux mesurés en Améd'autres pays, l'apport moyen d'acide rique du Nord, en Australie et au Japon, et l'un des plus bas en Europe, en raison d'une faible consommation des sources riches (huiles de colza, de soja, margarines). En termes d'adéquation des apports, la population française adulte est loin des actuelles recommandations : un apport en acide $\alpha$-linolénique égal à 0,8\% de l'apport énergétique, avec un rapport linoléique/a-linolénique aussi proche que possible de 5 [6] ou même moins [7]. De plus, si l'actuelle recommandation de diminuer I'apport lipidique de la ration est suivie $[6,41]$, il est vraisemblable qu'il en résultera une diminution concomitante de l'apport en acide $\alpha$-linolénique si rien n'est changé dans le choix des corps gras et/ou dans leur composition en acides gras.

En revanche, l'apport moyen en AGPI n-3 est relativement élevé, et l'apport en DHA satisfait l'apport recommandé pour $50 \%$ ou plus des sujets adultes. Cependant il y a de très grandes variations individuelles, dues aux grandes variations dans la consommation de poissons et de fruits de mer, les non-consommateurs n'atteignant en moyenne que la moitié environ de I'ANC du DHA. Encourager la consommation de poisson pour atteindre au moins deux portions par semaine, dont une portion de poisson gras, mais aussi promouvoir l'offre de produits enrichis en AGPI n-3 à longue chaîne, sont des moyens susceptibles d'améliorer la couverture des besoins en AGPI n-3, notamment en DHA, dans la population française.

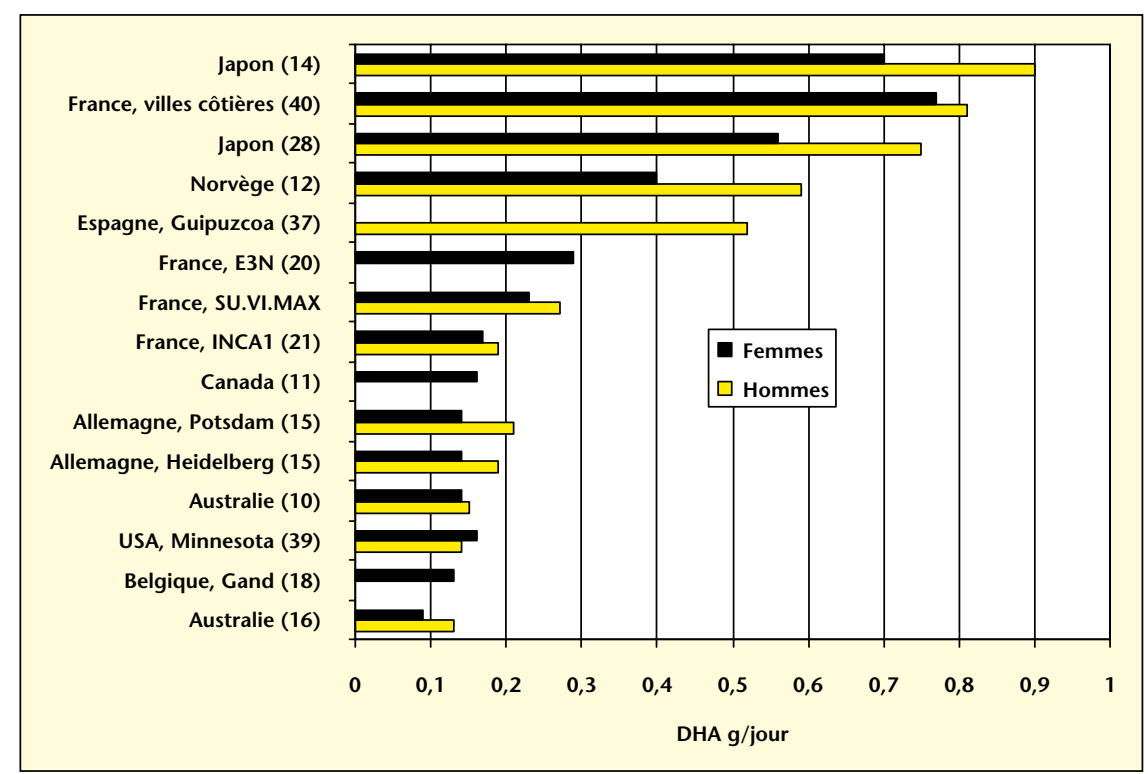

Figure 4. Consommation (g/jour) de DHA en Europe, aux Etats-Unis, en Australie et au Japon : études récentes sur la

Note : l'échantillon espagnol (37) est constitué d'adultes des deux sexes. 


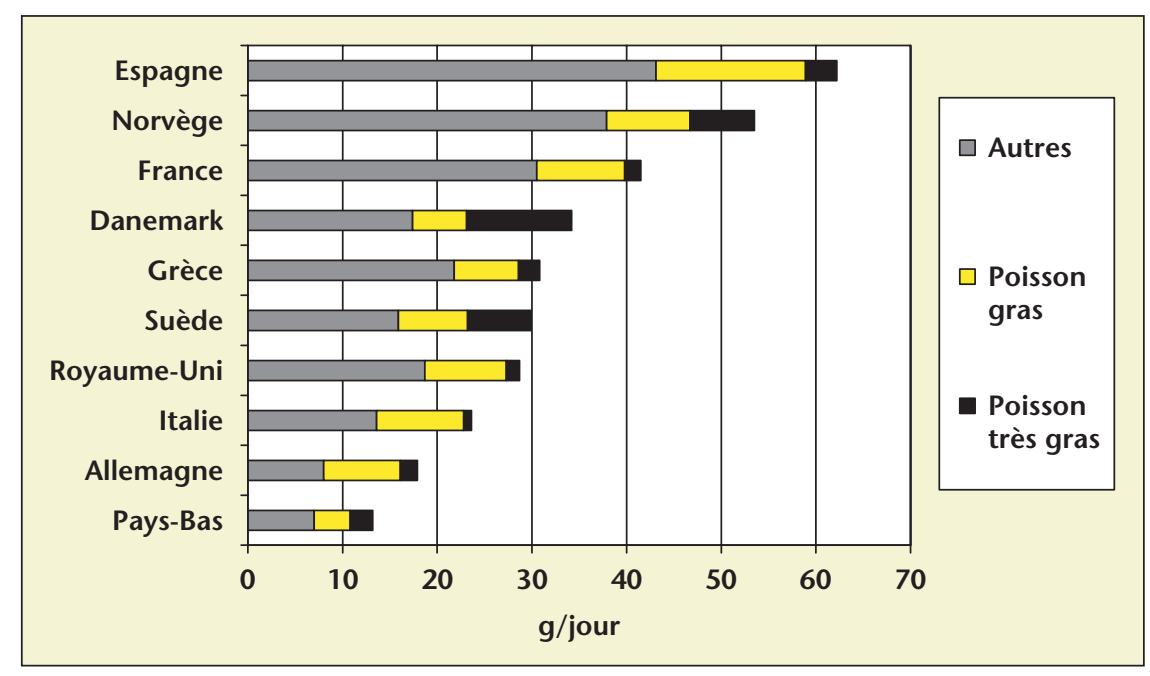

Figure 5. Consommation (g/jour) de poissons et produits de la mer chez les femmes de 10 pays d'Europe: multicohorte Epic (d'après Welch et al., réf. [38]).

Remerciements. L'auteur remercie M. Maillot et N. Darmon (UMR Inserm 476/Inra, Faculté de médecine de la Timone, Marseille) pour la communication de données originales non publiées issues de l'enquête INCA1.

\section{RÉFÉRENCES}

1. CARROLL DN, ROTH MT. Evidence for cardioprotective effects of omega- 3 fatty acids. Ann Pharmacother $2002 ; 36$ : 1950-6.

2. KRIS-ETHERTON PM, SHAFFER TAYLORD, YU-POTH S. Polyunsaturated fatty acids in the food chain in the United States. Am / Clin Nutr $2000 ; 71$ : 179S-189S.

3. SANDERS TAB. Polyunsaturated fatty acids in the food chain in Europe. Am / Clin Nutr 2000 ; 71 : 176S-178s.

4. SIMOPOULOS AP. The importance of the ratio of omega-6/omega-3 essential fatty acids. Biomed Pharmacother $2002 ; 56: 365-79$.

5. BURDGE GC. Metabolism of alpha-linolenic acid in humans. Prostaglandins Leukot Essent Fatty Acids $2006 ; 75$ : $161-8$.

6. LEGRAND $P$, BOURRE JM, DESCOMPS B, DURAND G, RENAUD S. Lipides. In : Martin A, ed. Apports nutritionnels conseillés pour la population française. $3^{e}$ édition. Paris: Tec \& Doc, $2001: 63-82$.

7. SIMOPOULOS AP, LEAF A, SALEM IR. N. Workshop on the Essentiality of and Recommended Dietary Intakes for Omega-6 and Omega-3 Fatty Acids. I Am Coll Nutr $1999 ; 18$ : 487-9.
8. COMBE N, BOUÉ C. Apports alimentaires en acides linoléique et alpha-linolénique d'une population d'Aquitaine. OCL $2001 ; 8: 118-21$.

9. DE VRIESE SR, DE HENAUW S, DE BACKER G, DHONT M, CHRISTOPHEAB. Estimation of dietary fat intake of Belgian pregnant women. Comparison of two methods. Ann Nutr Metab $2001 ; 45: 273-8$ Dietary intake of long-chain omega-3 polyunsaturated fatty acids: contribution of meat sources. Nutrition $2006 ; 22$ : 47-53.

11. INNIS SM, ELIAS SL. Intakes of essential n-6 and $n-3$ polyunsaturated fatty acids among pregnant Canadian women. Am / Clin Nutr $2003 ; 77: 473-8$.

12. JOHANSSON LR, SOLVOLL K, BJORNEBOE GE, DREVON CA. Intake of very-long-chain n-3 fatty acids related to social status and lifestyle. Eur / Clin Nutr 1998 ; 52 : 716-21.

13. KNUTSEN SF, FRASER GE, BEESON WL, LINDSTED KD, SHAVLIK DJ. Comparison of adipose tissue fatty acids with dietary fatty acids as measured by 24-hour recall and food frequency questionnaire in Black and White Adventists : the Adventist Health Study. Ann Epidemiol $2003 ; 13$ : 119-27.

14. KOBAYASHI M, SASAKI S, KAWABATA T, HASEGAWA K, TSUGANE $S$. Validity of a selfadministered food frequency questionnaire used in the 5-year follow-up survey of the JPHC Study Cohort I to assess fatty acid intake : compholipid level. J Epidemiol 2003 ; 13 : S64-S81.

15. LINSEISEN I, SCHULZE MB, SAADATIANELAHI M, KROKEA, MILLER AB, BOEING H. Quantity and quality of dietary fat, carbohydrate, and fiber intake in the German EPIC cohorts. Ann Nutr Metab 2003 ; 47 : 37-46.
10. HOWE P, MEYER B, RECORD S, BAGHURST K. parison with dietary records and serum phos-
16. MEYER BJ, MANN NJ, LEWIS JL, MILLIGAN GC, SINCLAIR AJ, HOWE PR. Dietary intakes and food sources of omega- 6 and omega- 3 polyunsaturated fatty acids. Lipids $2003 ; 38: 391-8$.

17. OKITAM, YOSHIDA S, YAMAMOTO I, et al. n-3 and n-6 fatty acid intake and serum phospholipid fatty acid composition in middle-aged women living in rural and urban areas in Okayama Prefecture. I Nutr Sci Vitaminol (Tokyo) $1995 ; 41: 313-23$.

18. SIOEN IA, PYNAERTI, MATTHYS C, DE BACKER G, VAN CAMP I, DE HENAUW S. Dietary intakes and food sources of fatty acids for Belgian women, focused on n-6 and n-3 polyunsaturated fatty acids. Lipids $2006 ; 41:$ 415-22.

19. HERCBERG S, PREZIOSI P, BRIANCON S, et al. A primary prevention trial using nutritional doses of antioxidant vitamins and minerals in cardiovascular diseases and cancers in a general population: the SU.VI.MAX study--design, methods, and participant characteristics. SUpplementation en Vltamines et Mineraux AntioXydants. Control Clin Trials 1998; 19: 336-51.

20. THIÉBAUT A. Etude et développement de méthodes pour l'analyse de données nutritionnelles recueillies dans le cadre d'enquêtes prospectives: application à la relation entre apport lipidique et cancer du sein. Thèse Université Paris-XI, 2004.

21. MAILLOT M, DARMON N. Communication personnelle. 2006.

22. Table de composition des aliments SUVIMAX. Paris : Editions Inserm, 2004.

23. FAVIER IC, IRELAND-RIPERT I, TOQUE C, FEINBERG M. Répertoire général des aliments, table de composition. 2e édition. Paris : Tec \& Doc, 1995.

24. IRELAND I, FAVIER JC, FEINBERG M. CIQUAL. Répertoire général des aliments. Tome 2: Produits laitiers. Paris : Tec \& Doc, 2002.

25. US DEPARTMENT OF AGRICULTURE, AGRICULTURAL RESEARCH SERVICE. USDA National Nutrient Database for Standard Reference, Release 16. 2003.

26. MINISTRY OF AGRICULTURE FISHERIES AND FOOD. Fatty Acids. Supplement to McCance \& Widdowson's The Composition of Foods. Cambridge : Royal Society of Chemistry. 1998.

27. KURIKI K, NAGAYAT, TOPKUDOME $Y$, et al. Plasma concentrations of n-3 highly unsaturated fatty acids are good biomarkers of relative dietary fatty acid intakes : a cross-sectional study. I Nutr $2003 ; 133$ : 3643-50.

28. OKUDA N, UESHIMA H, OKAYAMA A, et al. Relation of long chain n-3 polyunsaturated fatty acid intake to serum high density lipoprotein cholesterol among Japanese men in Japan and Japanese-American men in Hawaii : the INTERLIPID study. Atherosclerosis $2005 ; 178$ : 371-9. 
29. TOKUDOME Y, IMAEDA N, IKEDA M, KITAGAWA I, FUJIWARA N, TOKUDOME S. Foods contributing to absolute intake and variance in intake of fat, fatty acids and cholesterol in middle-aged Japanese. J Epidemiol 1999; 9 : 78-90.

30. TOKUDOME Y, KURIKI K, IMAEDA N, et al. Seasonal variation in consumption and plasma concentrations of fatty acids in Japanese female dietitians. Eur J Epidemiol $2003 ; 18$ : 945-53.

31. SUGANO M. Characteristics of fats in Japanese diets and current recommendations. Lipids 1996 ; 31 : S283-S286.

32. SUGANO M, HIRAHARA F. Polyunsaturated fatty acids in the food chain in Japan. Am / Clin Nutr $2000 ; 71$ : 189S-196S.

33. OLLIS TE, MEYER B], HOWE PR. Australian food sources and intakes of omega- 6 and omega-3 polyunsaturated fatty acids. Ann Nutr Metab $1999 ; 43$ : 346-55
34. VOSKUIL DW, FESKENS E], KATAN MB, KROMHOUT D. Intake and sources of alphalinolenic acid in Dutch elderly men. Eur / Clin Nutr $1996 ; 50$ : 784-7.

35. HULSHOF KFAM, VAN ERP-BAART MA, ANTTOLAINEN M, et al. Intake of fatty acids in Western europe with emphasis on trans fatty acids : the TRANSFAIR study. Eur / Clin Nutr $1999 ; 53$ : 143-57.

36. LINSEISEN ], BERGSTROM E, GAFA L, et al. Consumption of added fats and oils in the European Prospective Investigation into Cancer and Nutrition (EPIC) centres across 10 European countries as assessed by 24-hour dietary recalls. Public Health Nutr $2002 ; 5$ : 1227-42.

37. AMIANO $P$, DORRONSORO $M, D E$ RENOBALES M, RUIZ DE GORDOA JC, IRIGOYEN I, AND THE EPIC GROUP OF SPAIN. Very-long-chain omega- 3 fatty acids as markers for habitual fish intake in a population consuming mainly lean fish : the EPIC cohort of Gipuzkoa. Eur / Clin Nutr $2001 ; 55: 827-32$.
38. WELCH A, LUND E, AMIANO $P$, et al. Variability of fish consumtion within the 10 European countries participating in the European Investigation into Cancer and Nutrition (EPIC) study. Public Health Nutr $2002 ; 5$ : 1273-85.

39. MA J, FOLSOM AR, SHAHAR E, ECKFELDT JH, FOR THE ATHEROSCLEROSIS RISK IN COMMUNITIES (ARIC) STUDY INVESTIGATORS. Plasma fatty acid composition as an indicator of habitual dietary fat intake in middle-aged adults. Am J Clin Nutr 1995 ; 62 : 564-71.

40. LEBLANC JC, VOLATIER JL, SIROT V, BEMRAHAOUACHRIA N. Etude des consommations alimentaires de produits de la mer et imprégnation aux éléments traces, polluants et oméga-3. 2006 ; [Agence Française de Sécurité Sanitaire des Aliments (AFSSA)].

41. MINISTÈRE DE L'EMPLOI ET DE LA SOLIDARITÉ AND MINISTÈRE DÉLÉGUÉ À LA SANTÉ. Programme National Nutrition Santé 2001-2005. 2001. 